\title{
Validación de un instrumento para valorar la adherencia de pacientes al tratamiento hipertensivo
}

\author{
José Yamid Bolaños Cardozo MD. Mag, Dr. (C). \\ Docente - Investigador Grupo TECNNOSALUD. Escuela de Ciencias de la Salud. Universidad Nacional \\ Abierta y a Distancia.
}

Correspondencia: jose.bolanos@unad.edu.co

Recibido: 05/03/2014 Aceptado: 05/06/2014

\begin{abstract}
Resumen
Se validó un instrumento diseñado para medir la adherencia al tratamiento hipertensivo conformado por 10 ítems tipo Escala Likert. Se evaluó la validez de constructo mediante análisis factorial y confiabilidad por medio del Alpha de Cronbach. El análisis factorial permitió agrupar los ítems en torno a los factores que componen dicho constructo. El valor de Alfa obtenido $(0,515)$ corresponde a un valor moderado lo que significa que el instrumento puede ser utilizado como técnica adecuada de recolección de datos.
\end{abstract}

Palabras clave: confiabilidad, hipertensión, validez de contenido, validez de constructo.

\section{Validation of an instrument to assess patient adherence to antihypertensive therapy}

\begin{abstract}
An instrument was designed to measure the adhesion to the hypertension treatment using a tool composed of 10 items Likert-type scale. The construct validity through factorial analysis and reliability by using Cronbach's Alpha was evaluated. The factorial analysis allowed the grouping of the items around the factors that make up the construct. The alpha value obtained $(0.515)$ corresponds to a moderate value which means that the instrument can be used as a proper technique to collect data.
\end{abstract}

Keywords: construct validity, content validity, hypertension, reliability. 


\section{Introducción}

El control de la hipertensión arterial (HTA) es el principal objetivo en la prevención primaria y secundaria de los trastornos cardiovasculares (1). Uno de los factores determinantes en el inadecuado control de la enfermedad y la presencia de complicaciones es el escaso cumplimiento de las recomendaciones terapéuticas, lo cual se debe a un desinterés personal en la apropiada adherencia al plan de tratamiento (2-5).

Diferentes estudios informan que el tratamiento no farmacológico puede disminuir la presión arterial en un promedio de 11,4 a $5,5 \mathrm{~mm} \mathrm{Hg}$ en pacientes hipertensos y de 5,5 a $3 \mathrm{~mm} \mathrm{Hg}$ en individuos con presión arterial limítrofe, que es muy similar a las cifras que se obtienen con el tratamiento de algunos fármacos $(6,7)$.

La hipertensión como enfermedad crónica requiere cambios en el estilo de vida combinados o no con la toma de fármacos de forma permanente. La adherencia al tratamiento se puede definir como la incorporación de nuevos hábitos de vida unidos al cumplimiento del plan farmacológico, lo cual requiere motivación del paciente para el adecuado cumplimiento del plan terapéutico (8).

La percepción de la sintomatología por el paciente es uno de los factores predictores del seguimiento de las conductas terapéuticas instauradas por el grupo de salud a cargo (9-12).

\section{Materiales y métodos}

La investigación fue realizada en el período de mayo a septiembre de 2012. A partir de un estudio piloto previo, se seleccionó una muestra aleatoria del total de adultos hipertensos adscritos al programa de control de hipertensos en la Empresa Social del Estado (ESE) Centro de Salud Jorge González Olmos Páez del municipio de Páez en Boyacá, que corresponde a $(n=110)$ a quienes se les aplicó el instrumento de investigación.

\section{Elaboración del instrumento}

Para este efecto, se elaboró una Escala tipo Likert basada en el protocolo propuesto por Colás y Buendía (13) orientado a: definir la variable a medir que en la presente investigación corresponde al nivel de adherencia al tratamiento hipertensivo, formular los ítems o proposiciones acerca de la variable, seleccionar los ítems que más interesan eliminando los ambiguos, los que contienen una doble opinión y los que no miden lo mismo que los demás.

En cuanto a las alternativas de respuesta presentes en la escala, Canales (14) sugiere un mínimo de 3 y un máximo de 7.

Sin embargo, al respecto se consideraron factores como la edad y nivel educacional de los sujetos. Esto significa que es pertinente incluir un número reducido de opciones de respuesta en la escala a validar dirigida a los pacientes adultos, debido a que con su nivel educativo les resulta difícil discriminar entre muchos ítems. Por lo tanto, se consideró adecuado para los sujetos de la investigación en desarrollo el uso de tres opciones de respuesta: totalmente en desacuerdo, totalmente de acuerdo y parcialmente de acuerdo.

Para el cálculo de los resultados, debido a que la escala de Likert puede contener ítems positivos y negativos, se procedió de la siguiente forma:

a. A los ítems positivos se asignó las puntuaciones 1 y 3 , para totalmente en desacuerdo y totalmente de acuerdo, respectivamente.

b. Para los ítems negativos se invierten las puntuaciones asignando los valores de 3 para totalmente en desacuerdo y de 1 en el caso de totalmente de acuerdo.

Se realizó validación del instrumento, validez de contenido, confiabilidad del instrumento y validez del constructo, mediante los procedimientos estadísticos Coeficiente Alfa de Cronbach y Análisis Factorial, respectivamente $(15,16)$. 
Los factores obtenidos mediante análisis factorial fueron los siguientes:

El factor 1. Adherencia al tratamiento. Está referido al conocimiento y convicción de los beneficios de cumplir con las recomendaciones instauradas por el médico tratante en cuanto al aspecto farmacológico, dietético y de forma de vida. El factor se encontró conformado por los ítems:

P5: Conoce las complicaciones que puede ocasionar

la hipertensión arterial

P2: Cumple el tratamiento indicado por su médico

P1: Asiste a las consultas programadas por su médico para el seguimiento de la hipertensión arterial

P3: Cuando no cumple el tratamiento, se lo comunica a su médico y/o enfermera

El factor 2. Control y seguimiento. Se refiere al componente de evolución clínica de la hipertensión arterial de forma personalizada al paciente que está inscrito en el grupo de hipertensos en la ESE. Este factor está constituido por los ítems:
P9: Recibe de su médico y/o enfermera la información suficiente para comprender el tratamiento

P10: Recibe la educación por el médico y/o enfermera de forma individual y rara vez colectiva

P8: Se siente beneficiado (a) con el tratamiento o recomendación indicado

P7: Se siente vulnerable a las complicaciones de la hipertensión arterial

El factor 3. Complicaciones. Corresponde a la actividad previa a la ejecución del tratamiento farmacológico y luego de ser instaurado al paciente de manera permanente. Está constituido por este ítem:

P6: Siente la hipertensión arterial como una amenaza para su salud

\section{Resultados}

\section{Instrumento definitivo}

El instrumento definitivo, que se presenta a continuación, consta de 9 ítems con 3 alternativas de respuesta: totalmente de acuerdo, parcialmente de acuerdo, totalmente en desacuerdo, para ser administrado de forma individual en un tiempo promedio de 9 minutos.

Instrumento de conocimientos generales de tratamiento hipertensivo

Estimado (a) paciente hipertenso:

Con el objeto de fortalecerle su adherencia terapéutica, a continuación se presentan una serie de afirmaciones que debe contestar en concordancia a su criterio personal.

Por favor, marque con una $\mathrm{X}$ la respuesta que más se aproxime a su convicción.

Convenciones: TA: Totalmente de acuerdo; PA: Parcialmente de acuerdo; TD: Totalmente en desacuerdo

\section{$\mathbf{N}^{\circ}$}

Ítem

1 Conoce las complicaciones que puede ocasionar la hipertensión arterial

2 Cumple el tratamiento indicado por su médico

Asiste a las consultas programadas por su médico para el seguimiento de la hipertensión arterial

4 Cuando no cumple el tratamiento, se lo comunica a su médico y/o enfermera

5 Recibe de su médico y/o enfermera la información suficiente para comprender el tratamiento

6 Recibe la educación por el médico y/o enfermera de forma individual y rara vez colectiva

7 Se siente beneficiado (a) con el tratamiento o recomendación indicado

8 Se siente vulnerable a las complicaciones de la hipertensión arterial

9 Siente la hipertensión arterial como una amenaza para su salud 


\section{Discusión}

El presente estudio orienta las necesidades de mejorar las estrategias comunicativas de los profesionales de la salud, quienes pueden no dar el valor suficiente a una comunicación asertiva en la relación médico-paciente (17).

Este estudio buscó promover la investigación relacionada con intervención terapéutica basada en modelos integrales que transformen la percepción que tienen los pacientes acerca de la enfermedad y su tratamiento, debido a que la inexperiencia en el desarrollo del proceso salud-enfermedad conlleva al fracaso de la consecución de los objetivos terapéuticos $(18,19)$.

Según algunos estudios $(20,21)$, el instrumento de adherencia al tratamiento hipertensivo resulta ser muy útil para evaluar, de manera cuantitativa, la adherencia al tratamiento hipertensivo con un enfoque equivalente al de investigaciones de evaluación de material educativo y estrategias de educación, información y comunicación obtenidas en la literatura, debido a que el uso de este instrumento permitiría una evaluación más objetiva y concreta.

En un estudio reciente de Rodríguez, Molero y Acosta (22) realizado en 8862 pacientes se encuentra que el $31,70 \%$ no cumplía regularmente con el tratamiento instaurado y, entre las variables asociadas a la no adherencia terapéutica, se halló la "falta de síntomas» en el 28,85\% de los pacientes no adherentes. Es de anotar que la falta de adherencia al tratamiento hipertensivo por parte de algunos pacientes tiene como causa fundamental el relacionar cifras tensionales normales con la ausencia de sintomatología.

\section{Agradecimientos}

A los pacientes adultos hipertensos adscritos al programa de control de hipertensos en la Empresa Social del Estado (ESE) Centro de Salud Jorge
González Olmos Páez del Municipio de Páez. Este agradecimiento también se hace extensivo a la Dra. Luz Martha Vargas de Infante, directora del nodo ZCBOY- UNAD y al Dr. Pablo Alfonso Rodríguez Regalado, docente Universidad Privada Wiener.

\section{Referencias bibliográficas}

1. Ross, S., Walker, A. y MacLeod, M.J. Patient compliance in hypertension: Role of illness perceptions and treatment beliefs. Journal of Human Hypertension. 2004;18: 607-613.

2. Miller, N.H. Compliance with treatment regimens in chronic asymptomatic diseases. American Journal of Medicine. 1997; 102: 43-49.

3. Svensson, S., Kjellgren, K.L., Ahlner, J. y Saljo, R. Reason for adherence with antihypertensive medication. International Journal of Cardiology. 2000; 78: 157-163.

4. Thrall, G., Lip, G.Y.D. y Lane, D. Compliance with pharmacological therapy in hypertension: Can we do better, and how? Journal of Human Hypertension. 2004; 18: 595-597.

5. Wogen, J., Kreilick, C., Livornese, R.G. y Frech, F. A population-base $\mathrm{d}$ study of complianceand persistency with cardiovascular agents used in hypertension management. Journal of Hypertension. 2001; 4: 2-12A.

6. August P. Initial treatment of hypertension. N Engl J Med. 2003; 348 (7): 610-617.

7. Pescatello LS, Franklin BA, Fagard R, et al. American College of Sports Medicine. Position stand exercise and hypertension. Med Sci Sports Exerc. 2004; 36 (3): 533-553.

8. Luciano, M.C. y Herruzo, J. Some relevant components of adherence behavior. Journal of Behavior Therapy and Experimental Psychiatry. 1992; 23: 117-124.

9. Brondolo, E., Raymond, C., Rosen, P., John, B. y Kostis, M. Relationship of physical and mood to perceived and actual blood pressure in hypertensive men: A repeated-mesures desing. Psychosomatic Medicine. 1999; 61: 311-318.

10. Gil Roales-Nieto, J. Manual de Psicología de la Salud. Comportamiento y Salud/Enfermedad. Granada: Némesis. 2000 (2).

11. Leventhal, H., Meyer, D. y Nerenz, D. The commonsense representation of illness danger. En S. Rachman (Ed.), Contributions to medical psychology. Oxford: Pergamon Press.1980; 2: 7-30.

12. Visdómine, C. y Luciano. C. Locus de control y autorregulación conductual: revisiones conceptual y experimental. International Journal of Clinical and Health Psychology. 2006; 6: 729-751.

13. Colas, M. y Buendia, L. Investigación Educativa. Sevilla, España: Ediciones Alfar. 1998.

14. Canales, M. Metodología de investigación social. Introducción al oficio. Chile: LOM Ediciones. 2006.

15. Granados Gámez, G., Gil Roales-Nieto, J. e Ybarra Sagarduy, J.L. Un estudio exploratorio sobre el desarrollo de creencias sobre síntomas como señales de hipertensión arterial. Psicothema. 2006; 18: 822-827. 
16. Higgins, L.J. The associations of personal body awareness, symptom perception, and time line anticipation with adherence behavior of adults with primary hypertension. Doctoral

17. Hernández, R. Fernández, C. y Baptista, P. Metodología de la investigación. Editorial McGraw-Hill. 1996.

18. Haynes RB, Ackloo E, Sahota N, McDonald HP, Yao X. Interventions for enhancing medication adherence.

19. Ross S, Walker A, MacLeod MJ. Patient compliance in hypertension: Role of illness perceptions and treatment belief. J Hum Hypertens. 2004; 18: 607-13. http://dx.doi. org/10.1038/sj.jhh.1001721
20. Petrie KJ, Jago LA, Devcich DA. The rol of illness perceptions in patients of medical conditions. Curr Opin Psychiatry. 2007; 20: 163-7. http://dx.doi.org/10.1097/ YCO.0b013e328014a871

21. Rumbo JM, Arantón L, Goas UR, López N, Rivera P, Campos JM. Legibilidad de la documentación escrita en UCI. Neuquén: Federación Argentina de Cardiología; 2005. http:www. fac.org.ar/ccvc/llave/tl187/tl187.pdf

22. Rodríguez, H., Molero, M. y Acosta, L. Cumplimiento del tratamiento antihipertensivo en un área de salud. 2004. http://www.monografias.com/trabajos15/antihipertension/antihipertension.shtml. 